\title{
Armadilhas fotográficas na amostragem de mamíferos: considerações metodológicas e comparação de equipamentos
}

\author{
Ana C. Srbek-Araujo ${ }^{1} \&$ Adriano G. Chiarello ${ }^{1,2}$ \\ 1 Programa de Pós-Graduação em Zoologia de Vertebrados, Pontifícia Universidade Católica de Minas Gerais. \\ Avenida Dom José Gaspar 500, 30535-610 Belo Horizonte, Minas Gerais, Brasil. E-mail: srbekaraujo@hotmail.com; \\ bradypus@pucminas.com.br \\ 2 Autor correspondente.
}

\begin{abstract}
Use of camera traps in mammal sampling: methodological considerations and comparison of equipments. The use of camera traps is quite recent in Brazil. The high cost of equipments and constant expenses with supplies can, in some circumstances, limit the number of units to be deployed and sampling effort. The present study discusses methodological issues aiming at optimizing the use of these traps in mammalian inventories and also provides a comparison of two brands of equipments. The study was carried out in Santa Lúcia Biological Station (SLBS) in Center-north Espirito Santo state between January 2002 and November 2003, using three Wildlife Pro Camera ${ }^{\odot}$ (WPC) units and four DeerCam ${ }^{\odot}$ units. We realized the importance of sampling in different phitophysiognomies as they varied in mammal community. We detected a significant correlation between species richness and sampling effort in each trail, observing that $60 \%$ or more of species richness recorded in SLBS was obtained when sampling effort was $\geq 250$ camera-trap nights/trail. Nocturnal records predominated $(67 \%)$ although the number of recorded species was similar between night and day. When camera traps were set in pairs, only $27 \%$ of records were simultaneous. The tested units showed significant differences in durability and efficiency, both within and between brands.

KEY WORDS. Atlantic forest; inventories; mammalogy; methods; trapping.
\end{abstract}

RESUMO. A utilização de armadilhas fotográficas em estudos desenvolvidos no Brasil é recente. O alto custo dos equipamentos e os constantes gastos com material de consumo podem, em alguns casos, limitar o número de unidades e o tempo de amostragem. O presente estudo objetivou discutir questões metodológicas visando otimizar a utilização do equipamento no inventário de mamíferos, além de comparar dois modelos de armadiIhas fotográficas. $\mathrm{O}$ estudo foi desenvolvido na Estação Biológica de Santa Lúcia, no centro-norte do estado do Espírito Santo entre janeiro de 2002 e novembro de 2003, utilizando três Wildlife Pro Camera ${ }^{\odot}$ (WPC) e quatro DeerCam $^{\odot}$. Constatou-se a importância da realização de amostragens em diferentes fitofisionomias, tendo sido observadas variações na composição da comunidade registrada em cada ambiente. Foi detectada uma correlação significativa entre número de espécies registradas e esforço de amostragem empregado em cada trilha, ressaltando o registro de $60 \%$ ou mais dos táxons amostrados em trilhas com esforço $\geq 250$ armadilhas-dia. Houve predomínio de registros noturnos $(67 \%)$, embora o número de espécies registradas durante a noite tenha sido semelhante ao obtido ao longo do dia. Quando as armadilhas foram colocadas em pares, apenas $27 \%$ das fotografias foram simultâneas. Os equipamentos utilizados apresentaram diferenças significativas na durabilidade e eficiência na amostragem de mamíferos, tanto dentro como entre as marcas testadas.

PALAVRAS-CHAVE. Armadilhamento; Floresta Atlântica; inventários; mastozoologia; métodos.

A utilização de armadilhas fotográficas em estudos desenvolvidos no Brasil pode ser considerada recente, sendo empregadas na amostragem qualitativa da mastofauna (MARQUES \& Ramos 2001, Santos-Filho \& Silva 2002, Silveira et al. 2003, Trolle 2003a, b, Alves \& Andriolo 2005, Srbek-Araujo \& Chiarello 2005), na realização de estudos populacionais (Trolle \& Kéry 2003, 2005, Soisalo \& CaVALCANTI 2006) e como ferramenta com- plementar na obtenção de dados ecológicos (Santos-FilHo \& Silva 2002, Trolle 2003b, JÁcomo et al. 2004, Alves \& Andriolo 2005, Miranda et al. 2005, GaletTi et al. 2006).

CUTLER \& SWANN (1999) ressaltam que as armadilhas fotográficas são equipamentos sub-utilizados, particularmente em estudos que objetivam o inventariamento da fauna de vertebrados, a investigação da distribuição de espécies e nos que

Revista Brasileira de Zoologia 24 (3): 647-656, setembro 2007 
adotam modelos de captura-recaptura. Segundo Jennelle et al. (2002), a utilização de armadilhas fotográficas estaria melhor relacionada à definição da presença ou ausência de espécies em uma determinada área, recomendando o uso de modelos de captura-recaptura (estimativas populacionais e de densidade) apenas para espécies cujos indivíduos podem ser reconhecidos.

$\mathrm{O}$ armadilhamento fotográfico tem sido discutido quanto a melhor aplicação dos dados obtidos por este método (CARbone et al. 2001, 2002, Jennelle et al. 2002) e as vantagens e desvantagens deste, em comparação com métodos tradicionais de estudo, têm sido apresentadas por alguns autores (CUTLER \& Swann 1999, Silveira et al. 2003, SRbek-Araujo \& Chiarello 2005). Em estudo recente, Srbek-Araujo \& Chiarello (2005) demonstraram a eficiência das armadilhas fotográficas no inventário de mamíferos de médio e grande porte em áreas florestadas neotropicais, fornecendo resultados satisfatórios em longo prazo, corroborando com Voss \& Emmons (1996) ao considerar o caráter parcial ou complementar do método na amostragem da mastofauna, devendo ser utilizado em associação com outras técnicas para obtenção de dados da comunidade de mamíferos como um todo.

Considerando o alto custo do equipamento e os gastos com a manutenção dos estudos (baterias e filmes) e revelação/ ampliação dos negativos, que em muitos casos atuam como fator limitante do número de armadilhas fotográficas utilizadas e do tempo de amostragem, o presente estudo objetivou comparar a riqueza de espécies e o sucesso de amostragem obtido em diferentes fisionomias vegetacionais, trilhas e horários, visando auxiliar a adoção de desenhos amostrais mais eficientes e adequados à amostragem qualitativa da mastofauna em estudos futuros. De forma complementar, foi realizada uma análise comparativa de duas marcas/modelos de equipamentos importados disponíveis no mercado, visando a verificação de diferenças de durabilidade e eficiência na amostragem de mamíferos.

\section{MATERIAL E MÉTODOS}

\section{Área de Estudo}

As atividades de campo do presente estudo foram conduzidas na Estação Biológica de Santa Lúcia (EBSL), localizada entre as coordenadas $19^{\circ} 57^{\prime} 10^{\prime \prime}-19^{\circ} 59^{\prime} 00^{\prime \prime}$ S e $40^{\circ} 31^{\prime} 30^{\prime \prime}$ $40^{\circ} 32^{\prime} 25^{\prime \prime} \mathrm{W}$, no município de Santa Teresa, região serrana do estado do Espírito Santo. Atualmente, a EBSL é administrada pelo Museu de Biologia Professor Mello Leitão (MBML), localizado em Santa Teresa (ES), e pelo Museu Nacional, sediado no Rio de Janeiro (RJ). A Estação está inserida em uma das áreas de extrema importância biológica entre as Áreas Prioritárias para a Conservação da Biodiversidade da Mata Atlântica (Ministério do Meio Ambiente 2000), possuindo uma área de aproximadamente 440 ha de Mata Atlântica de encosta oficialmente protegidos (Mendes \& Padovan 2000). Considerando áreas vizinhas florestadas, o bloco de vegetação nativa no qual está inserida a EBSL alcança cerca de 900 ha.
A EBSL está situada no domínio geomorfológico Borda Montanhosa do Planalto, em altitudes que variam entre 550 e 950 m (Mendes \& Padovan 2000). De acordo com a classificação de Köppen, o clima na Estação é do tipo Cfa (subtropical úmido, sem estiagem), sendo caracterizado por apresentar uma estação quente e úmida, que se estende de outubro a março, e outra fria e seca, entre abril e setembro, com uma média pluviométrica anual de 1.868 mm (Mendes \& Padovan 2000). Thomaz \& Monteiro (1997) indicam uma temperatura média anual de $19,9^{\circ} \mathrm{C}$, estando a média das máximas em torno de $26,2^{\circ} \mathrm{C}$ e das mínimas próximo a $14,3^{\circ} \mathrm{C}$. A EBSL está sobre influência direta do Rio Timbuí (Bacia Hidrográfica do Rio Reis Magos), que atravessa o município de Santa Teresa, onde recebe dejetos domésticos, e percorre a Estação no sentido noroeste/sudeste, acolhendo um rico conjunto de córregos que nascem no interior da reserva (Mendes \& Padovan 2000).

A vegetação predominante na EBSL é do tipo Floresta Pluvial Atlântica Montana e Sub-Montana (PASsAmani 2000), estando enquadrada na região fitoecológica de Floresta Ombrófila Densa (Ibge 1993). De acordo com Mendes \& Padovan (2000), a cobertura vegetal é predominantemente florestal, havendo comunidades vegetais herbáceo-arbustivas em afloramentos rochosos e clareiras naturais. O levantamento florístico realizado por Thomaz \& Monteiro (1997) revelou a presença de 66 famílias, 178 gêneros e 476 espécies vegetais, sendo 443 arbóreas (perímetro à altura do peito $\geq 20 \mathrm{~cm}$ ), demonstrando uma diversidade de espécies superior à detectada em outros estudos realizados em áreas de Mata Atlântica. Os autores verificaram que a vegetação na EBSL encontra-se em um avançado estágio de sucessão, apresentando um baixo número de espécies características de locais perturbados (Thomaz \& Monteiro 1997). A riqueza de mamíferos não voadores registrada para Santa Teresa é igual ou maior do que a encontrada na maioria das áreas neotropicais, sendo superior à registrada em outras localidades já inventariadas de Mata Atlântica (PAssamani et al. 2000). Esta diversidade está representada por oito ordens, 22 famílias e 64 espécies, correspondendo a aproximadamente $40 \%$ das espécies de mamíferos não-voadores registradas para Mata Atlântica, apresentando $26 \%$ das consideradas endêmicas para o Bioma (PASSAMANI et al. 2000, SRbek-Araujo \& Chiarello 2005).

\section{Coleta de dados}

A investigação da mastofauna presente na EBSL através de armadilhas fotográficas foi realizada no período de janeiro de 2002 a novembro de 2003, totalizando aproximadamente 22 meses de estudo. Ao longo do período de amostragem foram utilizadas três armadilhas fotográficas modelo Wildlife Pro Camera $^{\circledR}$ (WPC), comercializadas pela Forest Suplies Inc. ${ }^{\odot}$ (EUA; este modelo é também vendido comercialmente com o nome de Camtrak $^{\odot}$ ou CamTrakker $^{\odot}$ ), e quatro armadilhas fotográficas modelo DeerCam - Scouting Camera (DC) ${ }^{\odot}$, fabricadas pela Non Typical Inc. ${ }^{\odot}$ (EUA). Os dois modelos empregados apresentam uma câmera fotográfica automática $35 \mathrm{~mm}$ e um sensor passivo (composto por uma única unidade produtora e receptora de luz 
infravermelho/cone de captação), projetado para detecção de calor e/ou movimento. Os equipamentos foram mantidos em funcionamento por 24 horas/dia no decorrer de todo o período de amostragem e ajustados para utilização do intervalo mínimo entre fotografias, de acordo com particularidades de cada modelo empregado (20 e 30 segundos para Wildlife Pro Camera e DeerCam, respectivamente). Foram utilizados filmes fotográficos coloridos FUJI ASA 200, 36 exposições, para cópias em papel, ao longo de todos os meses de amostragem.

As armadilhas fotográficas foram instaladas em trilhas antigas ( $n=9$; Bonita, Divisa D, Divisa E, Indaiaçu, Palmito, Sagui, Seca, Tapinoã e Timbuí) ou recentemente abertas ( $\mathrm{n}=3$; dos Monos, Nova e dos Porcos), estando estas distribuídas por toda a reserva, totalizando 28 pontos de amostragem. Foram contempladas áreas de Mata Primária, Mata Secundária e Capoeira, classificadas de acordo com feições da vegetação (grau de comunicação entre as copas e altura do dossel em relação ao solo, densidade do sub-bosque e do estrato herbáceo).

Objetivando o registro dos espécimes em sua distribuição natural, optou-se pela não utilização de iscas ou outros atrativos, uma vez que os indivíduos ou espécies podem reagir de forma diferenciada à presença de iscas (Cutler \& Swann 1999) e que sua utilização pode tornar a amostragem seletiva, aumentando o grau de detecção de determinadas espécies. Discussões relacionadas ao tipo de isca utilizada e as espécies registradas podem ser encontrados em Trolle (2003a).

Durante o período de amostragem foram adotadas duas abordagens metodológicas distintas, detalhadas a seguir.

Período 1. Em um primeiro momento (janeiro a novembro de 2002) foi realizado um estudo sistematizado para amostragem qualitativa das espécies de mamíferos presentes na EBSL, visando a realização de análises relacionadas à otimização do método. Durante este período foram utilizadas três armadilhas fotográficas modelo WPC. As armadilhas fotográficas foram instaladas em pontos escolhidos durante caminhadas ao longo das trilhas amostradas, tomando como parâmetros as características da vegetação (presença de espécimes em frutificação, sombreamento, etc), proximidade a corpos d'água, presença de rastros ou outros vestígios de atividade mastofaunística, entre outros. Os equipamentos eram vistoriados em intervalos regulares (aproximadamente 15 dias) para manutenção geral (renovação de filme fotográfico, bateria e pilhas, quando necessário; limpeza e verificação do estado de funcionamento do equipamento), sendo fixadas em árvores com diâmetro superior a 15 $\mathrm{cm}$, a aproximadamente $45 \mathrm{~cm}$ do solo. Tiras de elástico foram utilizadas para fixar o equipamento na posição considerada ideal, objetivando a obtenção de fotografias de boa qualidade e enquadramento, tendo sido utilizados também correntes e cadeados para evitar a perda de armadilhas. Quando o número e/ ou qualidade dos registros obtidos revelava-se muito pequeno (menos de uma fotografia a cada três dias), o equipamento era remanejado para outros pontos de amostragem. Durante o Período 1 foram realizadas amostragens em seis trilhas (Bonita, Di- visa D, Divisa E, Monos, Porcos e Tapinoã).

Período 2. Durante esta segunda abordagem (novembro de 2002 a novembro de 2003) foram utilizadas duas armadilhas fotográficas modelo WPC e quatro armadilhas fotográficas modelo DC objetivando a realização de análises comparativas dos equipamentos empregados. A partir do mapeamento das principais trilhas internas e limites da EBSL foram selecionados 18 pontos para instalação das câmeras, estando homogeneamente distribuídos na reserva (obedecendo a distância mínima de $300 \mathrm{~m}$ entre pontos). A sequência de amostragem dos pontos foi definida através de sorteio, realizando-se a vistoria das unidades empregadas em intervalos fixos de 30 dias para manutenção geral e relocação. Os equipamentos foram instalados observando-se os mesmos critérios adotados no primeiro ano, estando agrupados em pares compostos por unidades da mesma marca/modelo: um par de WPC e dois pares de DC (DC1 e DC2). A fixação das armadilhas foi semelhante ao adotado no período 1 , com exceção de que as duas armadilhas de cada par foram colocadas uma em cada lado da trilha, de maneira que ambas enquadrassem o mesmo ponto desta. Durante o posicionamento cuidado foi tomado para que o disparo de uma câmera (flash) não causasse o disparo da segunda câmera do par. A partir deste modelo objetivouse comparar a eficiência na amostragem das espécies de mamíferos, tanto entre equipamentos de uma mesma marca/modelo (comparação entre as unidades do par) quanto entre unidades de diferentes fabricantes (comparação entre os pares).

\section{Análise dos Dados}

Para as análises relacionadas à riqueza de espécies e número de registros obtidos em cada ambiente considerado (fisionomias vegetacionais) e nas trilhas amostradas durante $\mathrm{o}$ Período 1, foi considerado apenas um registro diário por espécie em cada ponto de amostragem. Para as análises relativas ao horário de obtenção dos registros (Período 1) e comparação de equipamentos (Período 2) foram considerados todos os registros, excluindo-se porém os registros consecutivos de uma mesma espécie, obtidos a partir de uma mesma armadilha fotográfica ou par de equipamentos, quando o intervalo entre as fotografias era inferior a cinco minutos. O intervalo de cinco minutos foi considerado suficiente para que o mesmo espécime, ou os indivíduos de um mesmo grupo social, não fossem contabilizados como dois ou mais registros de determinada espécie em uma única ocasião de captura, pois menos de $3 \%$ dos registros consecutivos da mesma espécie ocorreram entre 5 a 30 minutos de intervalo entre fotografias consecutivas.

A classificação do período de obtenção dos registros (diurno, noturno e crepuscular) foi estabelecida através do Programa Earthsun versão 4.6 (W. Scott Thoman, 830 Panel Road, Macedon, New York, USA). O programa utilizado considera dados astronômicos, longitude, latitude e altitude como base para o cálculo do horário exato de nascimento e pôr-do-sol, início e término do crepúsculo matutino e vespertino, para cada dia pesquisado, de acordo com a localidade.

Revista Brasileira de Zoologia 24 (3): 647-656, setembro 2007 
O esforço de captura foi definido por: [número de armadilhas fotográficas X número de dias de amostragem], onde cada dia corresponde a um período de 24 horas, e o sucesso de amostragem foi expresso em porcentagem, sendo calculado através da relação: [(número de registros/esforço de captura) X 100]. A contagem de registros para os dados obtidos durante o Período 2 foi realizada desconsiderando-se um dos registros efetuados simultaneamente para qualquer unidade do par de câmeras. Desta forma, o esforço de captura foi dado por: [número de pares de armadilhas fotográficas X número de dias de amostragem], onde cada par de equipamentos foi considerado uma unidade amostral. Isto se deve ao fato dos dois equipamentos cobrirem a mesma área e apresentarem igual probabilidade de captura dos espécimes a serem registrados em cada ponto. Não foi estabelecida alteração para o cálculo do sucesso de amostragem referente à amostragem realizada durante o Período 2 .

A verificação da ocorrência de diferenças significativas entre o número de registros obtido e esperado em cada fitofisionomia considerada, em função do esforço amostral empregado (câmeras-dia), foi realizada através do Teste do Qui-quadrado $\left(\chi^{2}\right)$. Testes semelhantes foram empregados para análise dos registros obtidos nas trilhas amostradas. O Teste de Correlação de Spearman foi empregado na verificação de correlações entre o número de espécies registradas e o esforço de amostragem realizado em cada trilha. Os dados de captura em função do período do dia (diurno e noturno, excluindo-se os classificados como crepuscular) também foram analisados através do Teste do Quiquadrado $\left(\chi^{2}\right)$. Este mesmo teste foi empregado ainda na verificação da ocorrência de diferenças significativas para o número de registros e pareamento de registros obtidos por cada par de armadilhas fotográficas e entre as unidades empregadas. A Correção de continuidade de Yates $\left[(\mathrm{O}-\mathrm{E})^{2}-0,5 / \mathrm{E}\right.$; onde $\mathrm{O}=$ freqüência observada e $E$ = freqüência esperada](ZAR 1996), foi adotada para os casos de grau de liberdade igual a um. Tendo em vista que os testes foram feitos manualmente, os valores de significância (p) são indicados em intervalos, após comparação com valores fornecidos em tabelas estatíticas (RoHLF \& SoKAL 1981). Para teste da eficiência dos equipamentos utilizados em relação às espécies amostradas, os registros obtidos foram agrupados em categorias de peso, definidas com base no peso médio das espécies fornecido por Emmons \& FeER (1997). Devido à imprecisão na identificação de algumas espécies de mamíferos de pequeno porte através de fotografias, optou-se pela identificação de determinados registros apenas no nível de Ordem (Didelphimorphia e Rodentia). A taxonomia e a nomenclatura das espécies amostradas foi estabelecida de acordo com WiLSON $\&$ ReEder (2005).

\section{RESULTADOS}

\section{Comparações entre ambientes e trilhas - Período 1}

Embora o número de registros tenha variado entre as três fisionomias vegetacionais amostradas (Tab. I), não foi observada diferença significativa para o número de registros obtidos nos três ambientes considerados (Teste do Qui-quadrado; $\chi^{2}=$ 0,045; g.l. = 2; p >0,05). O número total de espécies registradas também foi semelhante nos três ambientes (variação de 11 a 14 táxons), ressaltando que, embora o número de espécies amostradas na Mata Primária tenha sido igual ao detectado na Capoeira, a composição de espécies registradas em cada fitofisionomia foi diferenciada (Tab. I). Das 18 espécies amostradas no Período 1, sete foram registradas nos três ambientes, havendo oito registros coincidentes entre a Mata Primária e a Mata Secundária. Comparando-se a comunidade registrada na Capoeira com os registros obtidos nos ambientes florestais, verifica-se a presença de 13 espécies coincidentes entre aquele ambiente e os demais, sendo sete compartilhadas com ambas, três apenas com a Mata Primária e três com a Mata Secundária. Três táxons foram registrados exclusivamente na Mata Primária e um apenas na Capoeira. Não houve espécies registradas unicamente na Mata Secundária.

O esforço de amostragem realizado em cada trilha estudada variou de 10 a 259 câmeras-dia (Tab. II), estando localizados na Divisa (D) e Tapinoã os principais pontos de instalação dos equipamentos. Apesar disso, não foram observadas diferenças significativas no número de registros obtido entre trilhas quando diferenças no esforço amostral são levadas em conta no cálculo dos valores esperados (Teste do Qui-quadrado; $\chi^{2}=1,332$; g.l. $=5 ; \mathrm{p}>0,05)$. Ressalta-se, entretanto, que o número de espécies registradas esteve fortemente correlacionado ao esforço amostral empregado em cada trilha (Correlação de Spearman; rs $=0,943 ; \mathrm{n}=6 ; \mathrm{p}=0,005)$, destacando que $60 \%$ ou mais do total de espécies registradas durante o estudo foi obtido apenas nas trilhas que tiveram esforço $\geq 250$ armadilhas-dia.

\section{Horário de obtenção dos registros - Período 1}

Foram obtidos 191 registros de mamíferos ao longo do Período 1 (Tab. III), dos quais 104 apresentaram impressão nítida do dia e horário de realização da fotografia. Os 87 registros restantes $(45,6 \%)$ tiveram os dados comprometidos devido ao desajuste do equipamento nos primeiros meses de estudo ou a uma superexposição da fotografia no local onde os dados são impressos, tendo sido estes desconsiderados nas análises a seguir.

Foram detectadas diferenças significativas entre o número de registros obtido e esperado durante os períodos diurno (n $=30)$ e noturno $(n=60)$ (Teste do Qui-quadrado com correção de Yates; $\chi^{2}=9,978 ;$ g.l. $=1 ; p<0,01$ ), destacando que 66,67\% dos registros considerados foram efetuados à noite. Apesar da diferença no número de registros efetuados, o número de espécies registradas durante a noite foi semelhante ao obtido ao longo do dia $(\mathrm{n}=10)$, mas a composição da comunidade amostrada em cada período foi diferenciada. Embora tenha sido obtido um pequeno número de registros em horário crepuscular $(\mathrm{n}=14)$, foram amostradas nove espécies durante este período (50\% do total registrado), não tendo sido observados táxons registrados exclusivamente no crepúsculo. Quinze espécies foram fotografadas considerando os períodos diurno e crepuscular, enquanto 13 tiveram registros efetuados durante a noite e o crepúsculo. 
Tabela I. Espécies de mamíferos, número de registros (um por dia/câmera), esforço de amostragem (câmeras-dia), sucesso de captura e número de espécies registradas na Estação Biológica de Santa Lúcia, Santa Teresa (ES), durante o Período 1, de acordo com o ambiente amostrado. Os valores esperados com base no esforço amostral, são indicados entre parênteses.

\begin{tabular}{|c|c|c|c|c|}
\hline Espécies registradas & Mata primária & Mata secundária & Capoeira & Total \\
\hline Didelphis aurita (Wied-Neuwied, 1826) & 8 & 4 & 10 & 22 \\
\hline Metachirus nudicaudatus (É. Geoffroy, 1803) & 0 & 5 & 1 & 6 \\
\hline Cabassous sp. McMutrie, 1831 & 2 & 0 & 1 & 3 \\
\hline Dasypus novemcinctus Linnaeus, 1758 & 1 & 4 & 1 & 6 \\
\hline Cerdocyon thous (Linnaeus, 1766) & 0 & 1 & 1 & 2 \\
\hline Canis lupus familiaris Linnaeus, 1758 & 3 & 0 & 2 & 5 \\
\hline Leopardus pardalis (Linnaeus, 1758) & 1 & 3 & 6 & 10 \\
\hline Leopardus tigrinus (Schreber, 1775) & 2 & 0 & 0 & 2 \\
\hline Puma concolor (Linnaeus, 1771) & 0 & 1 & 2 & 3 \\
\hline Eira barbara (Linnaeus, 1758) & 1 & 1 & 1 & 3 \\
\hline Nasua nasua (Linnaeus, 1766) & 2 & 2 & 0 & 4 \\
\hline Procyon cancrivorus (G.[Baron] Cuvier, 1798) & 2 & 5 & 6 & 13 \\
\hline Mazama gouazoubira (G.Fischer [Von Waldhein], 1814)* & 3 & 0 & 2 & 5 \\
\hline Pecari tajacu (Linnaeus, 1758) & 1 & 2 & 1 & 4 \\
\hline Cuniculus paca (Linnaeus, 1766) & 2 & 4 & 1 & 7 \\
\hline Dasyprocta leporina (Linnaeus, 1758) & 17 & 0 & 0 & 17 \\
\hline Sciurus aestuans Linnaeus, 1766 & 3 & 0 & 0 & 3 \\
\hline Sylvilagus brasiliensis (Linnaeus, 1758) & 0 & 0 & 4 & 4 \\
\hline Didelphimorphia (não identificado) & 1 & 1 & 0 & 2 \\
\hline Rodentia (não identificado) & 2 & 2 & 0 & 4 \\
\hline Total de registros & 51 & 35 & 39 & 125 \\
\hline Total de registros esperado & 44,19 & 40,86 & 39,95 & 125 \\
\hline Esforço de amostragem & 292 & 270 & 264 & 826 \\
\hline Sucesso de captura (\%) & 17,5 & 13,0 & 14,8 & 23,1 \\
\hline Total de Espécies & 14 & 11 & 14 & 18 \\
\hline
\end{tabular}

* Em estudo anterior (SRbek-Araujo \& Chiarello 2005), esta espécie havia sido identificada como M. americana (Erxleben, 1777). Entretanto, análises posteriores à publicação citada indicaram que M. gouazoubira foi a espécie fotografada na EBSL.

\section{Equipamentos Empregados - Período 2}

Das seis armadilhas fotográficas empregadas durante o Período 2, três unidades foram desativadas no decorrer do estudo como consequência de falhas eletrônicas. Dois equipamentos DeerCam foram desativados após o sexto mês de amostragem, com um terceiro equipamento desativado após o oitavo mês.

Dos 136 registros efetuados durante o Período 2 (Tab. III), 132 foram obtidos através de armadilhas pareadas (quatro foram realizados após a desativação de uma das câmeras par). Considerando os dois modelos testados, apenas 26,5\% dos registros do período foram simultâneos e grande variação foi obtida, tanto entre marcas como entre pares da mesma marca (Tab. IV). O número de registros, respeitando diferenças no esforço amostral, foi significativamente diferente entre as duas câmeras do par DC1 e entre as duas câmeras do par DC2 (Teste do Qui-quadrado com correção de Yates; $\chi^{2}=23,200$ e 44,659, respectivamente; g.l. $=1 ; \mathrm{p}<0,001$ nos dois casos) (Tab. IV).
Para o par WPC não houve diferença significativa entre o número de registros obtidos por cada unidade do par (Teste do Qui-quadrado com correção de Yates; $\chi^{2}=0,656$; g.l. $=1 ; \mathrm{p}>$ 0,05) (Tab. IV).

O número de registros obtidos e o sucesso de amostragem foram diferenciados entre os três pares de câmera, tendo sido muito superiores para o Par WPC, em comparação com os outros pares de equipamentos (Tab. IV). Valores semelhantes são observados mesmo quando os dois pares DC foram analisados em conjunto (resultando em um esforço de amostragem superior) (Tab. IV). Considerando apenas os seis primeiros meses de amostragem, quando o esforço de amostragem foi semelhante para os três conjuntos, o número total de registros obtidos por par de equipamentos variou significativamente entre os três conjuntos (Teste do Qui-quadrado; $\chi^{2}=14,269$; g.l. $=2$; $\mathrm{p}<$ $0,001)$ (Tab. IV). Estes valores foram também significativos quando analisados isoladamente o Par WPC (Teste do Qui-qua- 
Tabela II. Espécies de mamíferos, número de registros (um por dia/câmera), esforço de amostragem (câmeras-dia), sucesso de captura, número e \% do total de espécies registradas na Estação Biológica de Santa Lúcia, Santa Teresa (ES), durante o Período 1, de acordo com a trilha amostrada. Os valores esperados com base no esforço amostral são indicados entre parênteses.

\begin{tabular}{|c|c|c|c|c|c|c|c|}
\hline Espécies amostradas & Bonita & Divisa (E) & Monos & Porcos & Tapinoã & Divisa (D) & Total \\
\hline Didelphis aurita & 0 & 0 & 1 & 3 & 10 & 8 & 22 \\
\hline Metachirus nudicaudatus & 0 & 0 & 1 & 4 & 1 & 0 & 6 \\
\hline Cabassous sp. & 0 & 0 & 0 & 0 & 1 & 2 & 3 \\
\hline Dasypus novemcinctus & 0 & 1 & 0 & 4 & 1 & 0 & 6 \\
\hline Cerdocyon thous & 0 & 0 & 1 & 0 & 1 & 0 & 2 \\
\hline Canis lupus familiaris & 0 & 0 & 1 & 0 & 1 & 3 & 5 \\
\hline Leopardus pardalis & 0 & 0 & 3 & 0 & 6 & 1 & 10 \\
\hline Leopardus tigrinus & 0 & 0 & 0 & 0 & 0 & 2 & 2 \\
\hline Puma concolor & 0 & 0 & 1 & 0 & 2 & 0 & 3 \\
\hline Eira barbara & 0 & 0 & 0 & 1 & 1 & 1 & 3 \\
\hline Nasua nasua & 0 & 1 & 0 & 2 & 0 & 1 & 4 \\
\hline Procyon cancrivorus & 0 & 2 & 0 & 5 & 6 & 0 & 13 \\
\hline Mazama gouazoubira & 0 & 3 & 0 & 0 & 2 & 0 & 5 \\
\hline Pecari tajacu & 0 & 0 & 0 & 2 & 1 & 1 & 4 \\
\hline Cuniculus paca & 0 & 5 & 0 & 0 & 1 & 1 & 7 \\
\hline Dasyprocta leporina & 0 & 0 & 0 & 0 & 0 & 17 & 17 \\
\hline Sciurus aestuans & 0 & 0 & 0 & 0 & 0 & 3 & 3 \\
\hline Sylvilagus brasiliensis & 0 & 0 & 0 & 0 & 4 & 0 & 4 \\
\hline Didelphimorphia (não identificado) & 0 & 0 & 1 & 0 & 0 & 1 & 2 \\
\hline Rodentia (não identificado) & 0 & 0 & 1 & 1 & 0 & 2 & 4 \\
\hline Total de registros & $0(1,51)$ & $12(8,17)$ & $10(14,68)$ & $22(22,55)$ & $38(38,89)$ & $43(39,19)$ & 125 \\
\hline Esforço de amostragem & 10 & 54 & 97 & 149 & 257 & 259 & 826 \\
\hline Sucesso de captura (\%) & 0 & 22,2 & 10,3 & 14,8 & 14,8 & 16,6 & 15,1 \\
\hline Total de espécies & 0 & 5 & 6 & 7 & 14 & 11 & 18 \\
\hline$\%$ do total de espécies & 0 & 27,8 & 33,3 & 38,9 & 77,8 & 61,1 & 1 \\
\hline
\end{tabular}

drado com correção de Yates; $\chi^{2}=9,140 ;$ g.l. = 1 ; $\left.\mathrm{p}<0,01\right)$ e o Par DC2 (Teste do Qui-quadrado com correção de Yates; $\chi^{2}=$ 4,414; g.l. = $1 ; \mathrm{p}<0,05)$. Para o par DC1 as diferenças não foram significativas (Teste do Qui-quadrado com correção de Yates; $\chi^{2}=0,829 ;$ g.l. $\left.=1 ; \mathrm{p}>0,05\right)$.

$\mathrm{O}$ número de espécies registradas foi semelhante entre o Par WPC e DC1 (10 e 9 táxons, respectivamente), com um valor inferior registrado para o Par DC2 (apenas seis espécies) (Tab. IV). Agrupando as espécies registradas em três categorias de peso (<1 kg; 1-10 kg e > 10 kg) (Fig. 1), observou-se uma similaridade na frequência de registros obtidos para cada categoria entre os pares WPC, DC1 e DC2 (Teste do Qui-quadrado; $\chi^{2}=4,611$; g.l. $=4 ; \mathrm{p}>0,10)$. O resultado do teste se mantém não significativo $\left(\chi^{2}=4,319 ;\right.$ g.l. $\left.=2 ; \mathrm{p}>0,10\right)$ também quando a primeira categoria de peso $(<1 \mathrm{~kg})$ é excluída da análise, já que esta apresenta freqüência esperada igual a zero em um dos casos, o que torna problemática a aplicação do teste do Qui-quadrado (ZAR 1996).

Analisando os registros obtidos ao longo do período de amostragem, verificou-se que apesar da baixa taxa de ocorrência de registros simultâneos (26,5\%), houve variações na eficiência do pareamento das unidades de acordo com as espécies registradas (tamanho corporal) e com a marca/modelo de equipamento empregado. Apenas a onça-parda [Puma concolor (Linnaeus, 1771)], espécie de grande porte, foi fotografada simultaneamente pelas duas unidades de cada par dos três pares usados. A onça-parda foi a única espécie com registros simultâneos nos pares de DC. Por sua vez, o par de WPC obteve registros simultâneos para as seguintes espécies: Dasyprocya leporina (Linnaeus, 1758) (cutia), Canis lupus familiaris Linnaeus, 1758 (cão doméstico), Leopardus pardalis (Linnaeus, 1758) (jaguatirica), Nasua nasua (Linnaeus, 1766) (quati), Mazama gouazoubira (G.Fischer [Von Waldhein], 1814) (veado-catingueiro), Pecari tajacu (Linnaeus, 1758) (caititu) e Dasypus novemcinctus Linnaeus, 1758 (tatu-galinha). O número de registros pareados obtidos durante os seis primeiros meses de amostragem foi 15 e cinco vezes maior para o conjunto WPC em relação aos Pares DC1 e DC2, respectivamente.

Com relação ao horário de obtenção dos registros (crepúsculo, diurno e noturno), não houve associação significativa entre tipo de equipamento (DC x WPC) e horário do registro (Teste do Qui-quadrado; $\chi^{2}=0,134 ;$ g.l. $=2 ; \mathrm{p}>0,90$ ) (Fig. 

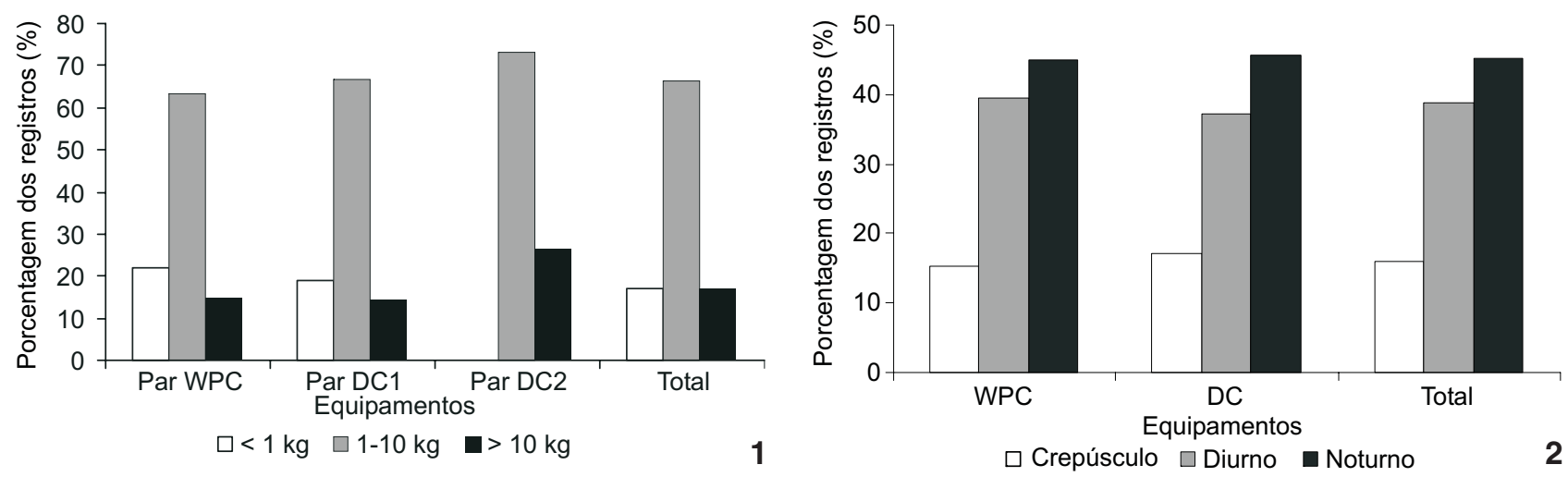

Figuras 1-2. (1) Porcentagem de registros de mamíferos obtidos na Estação Biológica de Santa Lúcia, Santa Teresa (ES), para cada par de equipamentos empregados durante os seis primeiros meses do Período 2 (novembro de 2002 a novembro de 2003), de acordo com a categoria de peso das espécies registradas; (2) Comparação entre a porcentagem de registros de mamíferos obtidos na Estação Biológica de Santa Lúcia, Santa Teresa (ES), para cada marca/modelo de equipamentos empregados durante o Período 2 (novembro de 2002 a novembro de 2003), de acordo com o horário de obtenção dos registros. Ver seção Material e Métodos para detalhes.

Tabela III. Número de registros (cinco minutos de intervalo), esforço de amostragem e sucesso de captura obtido na Estação Biológica de Santa Lúcia, Santa Teresa (ES) durante os dois períodos de estudo. Ver seção Material e Métodos para detalhes.

\begin{tabular}{|c|c|c|c|}
\hline Espécies & Período 1 & Período 2 & Total \\
\hline Cuniculus paca & 8 & 10 & 18 \\
\hline Cabassous sp. & 3 & 2 & 5 \\
\hline Canis lupus familiaris & 8 & 27 & 35 \\
\hline Cerdocyon thous & 2 & 4 & 6 \\
\hline Dasyprocta leporina & 45 & 16 & 61 \\
\hline Dasypus novemcinctus & 11 & 9 & 20 \\
\hline Didelphis aurita & 34 & 12 & 46 \\
\hline Eira barbara & 3 & 0 & 3 \\
\hline Euphractus sexcinctus (Linnaeus, 1758) & 0 & 1 & 1 \\
\hline Hydrochoerus hydrochaeris (Linnaeus, 1766) & 0 & 1 & 1 \\
\hline Leopardus pardalis & 10 & 15 & 25 \\
\hline Leopardus tigrinus & 2 & 0 & 2 \\
\hline Mazama gouazoupira & 8 & 6 & 14 \\
\hline Metachirus nudicaudatus & 12 & 8 & 20 \\
\hline Nasua nasua & 7 & 5 & 12 \\
\hline Pecari tajacu & 4 & 4 & 8 \\
\hline Philander frenatus (Olfers, 1818) & 0 & 1 & 1 \\
\hline Procyon cancrivorus & 16 & 2 & 18 \\
\hline Puma concolor & 3 & 7 & 10 \\
\hline Sciurus aestuans & 3 & 1 & 4 \\
\hline Sylvilagus brasiliensis & 6 & 1 & 7 \\
\hline Didelphimorphia (não identificado) & 2 & 0 & 2 \\
\hline Rodentia (não identificado) & 4 & 4 & 8 \\
\hline Total de registros & 191 & 136 & 327 \\
\hline Esforço de amostragem & 826 & 900 & 1726 \\
\hline Sucesso de captura (\%) & 23,12 & 15,11 & 18,95 \\
\hline Total de espécies & 18 & 19 & 21 \\
\hline
\end{tabular}


Tabela IV. Número de registros, número de espécies, esforço de amostragem (câmeras-dia) e sucesso de captura obtido na Estação Biológica de Santa Lúcia, Santa Teresa (ES), para cada par de equipamentos empregado durante o Período 2. Os valores relativos aos seis primeiros meses de estudo são mostrados entre parênteses. "A" e "B" denotam as duas unidades de cada par de câmeras. Ver seção Material e Métodos para detalhes.

\begin{tabular}{|c|c|c|c|c|c|c|c|c|}
\hline & \multirow{2}{*}{$N^{\circ}$ Registros } & \multirow{2}{*}{ No Espécies } & \multirow{2}{*}{ Esforço } & \multirow{2}{*}{ Sucesso } & \multicolumn{2}{|c|}{ Unidades } & \multicolumn{2}{|c|}{ Registros simultâneos } \\
\hline & & & & & $A$ & B & $\mathrm{N}$ & $(\%)$ \\
\hline Par WPC & $91(41)$ & $15(10)$ & 360 & 25,28 & 66 & 57 & $31(15)$ & 34,07 \\
\hline Par DC1 & $22(22)$ & $9(9)$ & 180 & 12,22 & 9 & 14 & $1(1)$ & 4,55 \\
\hline Par DC2 & $19(15)$ & $9(6)$ & 240 & 7,92 & 7 & 15 & $3(3)$ & 15,79 \\
\hline \multirow[t]{2}{*}{ Total DC } & $41(37)$ & $14(11)$ & 420 & 9,76 & - & - & $4(4)$ & 9,76 \\
\hline & $132(78)$ & $19(17)$ & 780 & 16,92 & - & - & $35(19)$ & 26,52 \\
\hline
\end{tabular}

2). Ou seja, nos dois tipos de equipamentos o maior número de registros foi obtido no período noturno, seguido pelo período diurno e crepúsculo.

\section{DISCUSSÃO}

Os dois modelos de armadilhas fotográficas empregados revelaram-se resistentes às adversidades climáticas de temperatura e umidade locais (elevada ocorrência de chuvas na região). É importante destacar, entretanto, que foram realizadas adaptações nas DeerCam antes de sua instalação, tendo sido utilizados remendos de borracha e cola de silicone para vedação dos locais de comunicação entre o interior do equipamento e o meio externo (interruptor liga/desliga e janelas/visores). Foi também importante a realização de manutenção constante dos equipamentos empregados visando a remoção de fungos e impurezas do seu interior e superfície externa, garantindo seu funcionamento e acurácia. Observou-se também a presença de pequenos insetos e aranhas no compartimento externo do sensor nas Wildlife Pro Camera, que em alguns casos estava sendo utilizado para a construção de abrigos/ninhos. Deste modo, a falta de manutenção, especialmente em relação a fungos e umidade, pode comprometer o mecanismo óptico e/ou eletrônico do equipamento, corroborando CuTLER \& SWANN (1999). Em relação à ocorrência de falhas eletrônicas detectadas nos equipamentos, com a consequente desativação de unidades durante o período de estudo, os mesmos autores mencionam a existência de "problemas mecânicos crônicos" em armadilhas fotográficas utilizadas em vários estudos, destacando ser esta uma das desvantagens deste método de amostragem (CuTLer \& Swann 1999).

Embora o tempo de armadilhamento fotográfico (esforço de amostragem) executado durante o Período 2 tenha sido $9,0 \%$ superior ao Período 1 , um menor número de registros foi obtido no Período 2 (55 registros a menos) (Tab. III). Apesar da possibilidade de haver diferenças na composição da comunidade de mamíferos entre os diferentes ambientes e trilhas, é mais provável que a redução do sucesso de captura observada durante o Período 2 esteja principalmente relacionada à eficiência dos modelos de armadilhas fotográficas utilizadas nos dois momentos. A análise comparativa dos equipamentos em- pregados indicou uma inferioridade significativa das armadilhas fotográficas modelo DeerCam em relação às Wildlife Pro Camera, tanto em relação à obtenção de registros quanto à detecção das espécies. Vale ressaltar ainda que as DeerCam não foram eficientes na obtenção de registros simultâneos, não sendo portanto recomendadas em estudos nos quais objetiva-se a realização de investigações populacionais com o pareamento de equipamentos. É preciso ressaltar, entretanto, que como apenas um par do modelo Widlife Pro Camera foi usado, as diferenças apontadas entre as duas marcas devem ser apenas sugestivas, até que estudos futuros possam avaliar um maior número de câmeras. Ainda com relação à comparação de equipamentos, JACKSON et al. (2005) apresentam uma detalhada descrição comparativa de diferentes marcas/modelos de equipamentos (DeerCam, TrailMaster 1550 e 550, CamTrakker Original e Digital). Entretanto, como o objetivo do referido estudo foi o desenvolvimento de um protocolo para estudos com leopardos da neve, não foram apresentados dados comparativos da eficiência de cada equipamento em relação ao número de fotografias obtidas, detecção das espécies de maneira geral ou pareamento das unidades.

A amostragem em diferentes ambientes revelou-se necessária para a obtenção de um volume mais significativo de registros e de um maior número de espécies, ressaltando-se diferenças na composição da comunidade entre os ambientes amostrados, embora não tenha havido diferença significativa para o número absoluto de espécies registradas em cada fitofisionomia (Período 1). Destaca-se que algumas espécies foram registradas preferencialmente em determinado ambiente, reforçando que a realização de amostragens em ambientes diversificados pode favorecer a obtenção de registros mais representativos da comunidade como um todo. Os dados obtidos sugerem também a aplicabilidade das armadilhas fotográficas em estudos relacionados à investigação de preferências no uso de hábitats pelas espécies (meso-hábitat). O esforço de captura empregado foi muito diferenciado entre as trilhas amostradas no primeiro período de estudo. De forma semelhante ao detectado para os ambientes, sugere-se que, apesar de existir uma relação entre esforço de amostragem e sucesso de captura, fatores

Revista Brasileira de Zoologia 24 (3): 647-656, setembro 2007 
intrínsecos ao local de instalação do equipamento também podem influenciar na obtenção dos registros. Desta forma, os dados indicam a importância da realização de esforços de captura superiores a 250 câmeras-dia quando se objetiva a obtenção de registros de um maior número de espécies.

Não foram detectadas diferenças significativas para o número de táxons registrados nos períodos diurno ou noturno. Este fato pode estar associado ao registro de diferentes espécies nos dois períodos,de forma semelhante ao observado por GómEz et al. (2005). De forma semelhante, Silveira et al. (2003) consideraram que o equipamento é similarmente eficiente para a detecção de espécies noturnas e diurnas. Entretanto, deve-se ressaltar possíveis diferenças na detecção do calor corporal dos espécimes entre os períodos, já que houve maior numero de registros durante a noite. Considerando que em geral a temperatura ambiente durante o dia é superior à observada no período noturno, pode haver um favorecimento da obtenção de registros à noite, através de uma maior diferença entre temperaturas ambiente e corporal, com uma consequente melhoria na eficiência de detecção dos espécimes pelo equipamento (SRBEK-Araujo \& Chiarello 2005). Ainda neste sentido, considera-se também que a influência de diferenças de temperatura na eficiência de detecção dos grupos faunísticos pode contribuir com a obtenção de um menor volume de registros no período mais quente do dia, em associação com uma redução das atividades por parte das espécies no mesmo período. De forma semelhante, Trolle \& KéRY (2003) mencionam uma dificuldade do sensor em detectar a presença de espécies de sangue quente em áreas abertas quando a temperatura ambiente é alta.

De acordo com o apontado por Srbek-Araujo \& Chiarello (2005), a ausência de padronização entre os desenhos amostrais empregados dificulta a realização de comparações entre os dados obtidos em estudos realizados no Brasil. Acrescenta-se também que a ocorrência de diferenças na eficiência da amostragem de mamíferos entre marcas/modelos de equipamento, conforme apontado no presente estudo, pode ser um fator limitante na comparação entre estudos. A importância de investigações para comparação da eficiência entre equipamentos diferentes e das formas de instalação e utilização destes já haviam sido levantadas por CutLer \& Swann (1999).

Em síntese, o presente estudo demonstrou a importância: (1) da realização de amostragens em diferentes fitofisionomias e locais (trilhas) disponíveis em uma determinada área de estudo, (2) da manutenção das armadilhas fotográficas em funcionamento durante 24 horas/dia, e (3) do emprego de períodos de amostragem mais longos (não inferiores a 250 câmeras-dia), quando se objetiva a amostragem mais completa da comunidade de mamíferos de médio e grande porte. Além disso, o estudo demonstrou a importância da padronização de equipamentos quando se pretende comparar ambientes dentro de uma mesma localidade ou localidades entre si, visto que cada marca de armadilha fotográfica apresenta idiossincrasias quanto ao funcionamento e eficiência. Vale lem- brar, ainda, que esta heterogeneidade no funcionamento de armadilhas fotográficas dentro de um mesmo estudo, pode comprometer premissas que são fundamentais à correta estimativa de parâmetros demográficos, como densidade e tamanho populacional, que envolvam protocolos de captura-marcação-recaptura (Karanth \& Nichols 1998, Trolle \& Kéry 2003, Soisalo \& Cavalcanti 2006).

\section{AGRADECIMENTOS}

Ao Museu de Biologia Prof. Mello Leitão, especialmente ao Diretor Helio de Queiroz B. Fernandes e à Secretária Rose Loss, pelo prestimoso apoio logístico; aos companheiros de campo -Eduardo Martins de Barros, Leandro Santana Moreira, Luciana Barçante, Marina Nogueira dos Santos, Oscar Echevery, Paula Lara Ruiz, Pedro Amaral, Rodrigo Lira Meyer, Sérgio Pimenta Costa e Valeska Buchemi de Oliveira - pelo auxílio na coleta de dados; ao CNPq pelo apoio financeiro (projeto "Biodiversidade da Mata Atlântica no Estado do Espírito Santo", Processo 469.321/2000-8) e bolsa de produtividade em pesquisa (AGC); ao Fundo de Incentivo à Pesquisa da PUC Minas (Processo FIP 2002/06 - TLE) pelo financiamento e CAPES pela bolsa de mestrado (ACSA).

\section{REFERÊNCIAS BIBLIOGRÁFICAS}

Alves, L.C.P.S. \& A. Andriolo. 2005. Camera traps use on the mastofaunal survey of Araras Biological Reserve, IEF-RJ. Revista Brasileira de Zoociências 7 (2): 231-246.

Carbone, C.; S. Christie; K. Conforti; T. Coulson; N. Franklin; J.R. Ginsberg; M. Griffiths; J. Holden; K. Kawanishi; M. Kinnaird; R. Laidlaw; A. Lynam; D.W. MacDonald; D. Martyr; C. McDougal; L. Nath; T.O. O'Brien; J. Seidensticker; D.J.L. Smith; M. Sunquist; R. Tilson \& W.N. wan Shahruddin. 2001. The use of photographic rates to estimate densities of tigers and other cryptic mammals. Animal Conservation 4: 75-79.

Carbone, C.; S. Christie; K. Conforti; T. Coulson; N. Franklin; J.R. Ginsberg; M. Griffiths; J. Holden; M. Kinnaird; R. Laidlaw; A. Lynam; D.W. MacDonald; D. Martyr; C. McDougal; L. Nath; T.O. O'Brien; J. SeIDENSTicker; D.J.L. SMith; R. Tilson \& W.N. WAN SHAHruddin. 2002. The use of photographic rates to estimate densities of tigers and other cryptic mammals: response to Jennelle et al. Animal Conservation 5: 121-132.

Cutler, T.L. \& D.E. Swann. 1999. Using remote photography in wildlife ecology: a review. Wildlife Society Bulletin 23 (3): 571-581.

EMmons, L.H. \& F. Feer. 1997. Neotropical Rainforest mammals. A field guide. Chicago, University of Chicago Press, $2^{\text {nd }}$ ed., $307 \mathrm{p}$.

Galetti, M.; C.I. Donatti; A.S. Pires; P.R. Guimarães JR. \& P. Jordano. 2006. Seed survival and dispersal of an endemic Atlantic forest palm: the combined effects of defaunation and forest fragmentation. Botanical Journal of the Linnean Society, London 151 (1): 141-149. 
Gómez, H.; R.B. Wallace; G. Ayala \& R. Tejada. 2005. Dry season activity periods of some Amazonian mammals. Studies on Neotropical Fauna and Environment 40 (2): 91-95

IвGe. 1993. Mapa de Vegetação do Brasil. Rio de Janeiro, Fundação Instituto Brasileiro de Geografia e Estatística, 1p.

JACKSON, R.M.; J.D. RoE; R. WANGChuK \& D.O. Hunter. 2005. Surveying snow leopard population with emphasis on camera trapping. A handbook. Sonoma, California, The Snow Leopard Conservancy, 73 p. Avalable in the World Wide Web at: http://www.snowleopardconservancy.org [accessed 07.IX.2006].

JÁcomo, A.T.A.; L. Silveira \& J.A.F. Diniz-Filho. 2004. Niche separation between the maned wolf (Chrysocyon brachyurus), the crab-eating fox (Dusicyon thous) and the hoary fox (Dusicyon vetulus) in central Brazil. Journal of Zoology 262 (1): 99-106.

Jennelle, C.S.; M.C. Runge \& D.I. Mackenzie. 2002. The use of photographic rates to estimate densities of tigers and other cryptic mammals: a comment on misleading conclusions. Animal Conservation 5: 119-120.

Karanth, K.U. \& J.D. Nichols. 1998. Estimation of tiger densities in India using photographic captures and recaptures. Ecology 79: 2852-2862.

Marques, R.V. \& F.M. Ramos. 2001. Identificação de mamíferos ocorrentes na Floresta Nacional de São Francisco de Paula/ Ibama, RS com a utilização de equipamento fotográfico acionado por sensores infravermelhos. Divulgações do Museu de Ciências e Tecnologia 6: 83-94.

Mendes, S.L. \& M.P.A. Padovan. 2000. Estação Biológica de Santa Lúcia, Santa Teresa, Espírito Santo. Boletim do Museu de Biologia Mello Leitão 11/12: 7-34.

Ministério do MeIo Ambiente. 2000. Avaliação e ações prioritárias para a conservação da biodiversidade da Mata Atlântica e Campos Sulinos. Brasília, MMA/SBF, 40p.

Miranda, J.M.D.; I.P. Bernardi; K.C. Abreu \& F.C. Passos. 2005. Predation on Alouatta guariba clamitans Cabrera (Primates, Atelidae) by Leopardus pardalis (Linnaeus) (Carnivora, Felidae). Revista Brasileira de Zoologia 22 (3): 793-795.

Passamani, M. 2000. Análise da comunidade de marsupiais em Mata Atlântica de Santa Teresa, Espírito Santo. Boletim do Museu de Biologia Mello Leitão 11/12: 215-228.

Passamani, M.; S.L. Mendes \& A.G. Chiarello. 2000. Non-volant mammals of the Estação Biológica de Santa Lúcia and adjacent areas of Santa Teresa, Espírito Santo, Brazil. Boletim do Museu de Biologia Mello Leitão 11/12: 201-214.

RoHLF, F.J. \& R.B. SoKAL. 1981. Statistical tables. New York, W.H. Freeman \& Company, $2^{\text {nd }}$ ed., VI+219p.

SAnTos-Filho, M. \& M.N.F. Silva. 2002. Uso de habitats por mamíferos em área de Cerrado do Brasil Central: um estudo com armadilhas fotográficas. Revista Brasileira de Zoociências 4 (1): 57-73.

Silveira, L.; A.T.A. JÁcomo \& J.A.F. DinIZ-Filho. 2003. Canera trap, line transect census and track surveys: a comparative evaluation. Biological Conservation 114 (3): 351-355.

Solsalo, M.K. \& S.M.C. CavalCanTI. 2006. Estimating the density of a jaguar population in the Brazilian Pantanal using cameratraps and capture-recapture sampling in combination with GPS radio-telemetry. Biological Conservation 129 (1): 487496.

Srbek-Araujo, A.C. \& A.G. Chiarello. 2005. Is camera-trapping an efficient method for surveying mammals in Neotropical forests? A case study in south-eastern Brazil. Journal of Tropical Ecology 21 (1): 121-125.

Thomaz, L.D. \& R. Monteiro. 1997. Composição florística da Mata Atlântica de encosta da Estação Biológica de Santa Lúcia, município de Santa Teresa - ES. Boletim do Museu de Biologia Mello Leitão 7: 3-48.

Trolle, M. 2003a. Mammal survey in the Rio Jauaperí region, Rio Negro Basin, the Amazon, Brazil. Mammalia 67 (1): 75 83.

Trolle, M. 2003b. Mammal survey in the southeastern Pantanal, Brazil. Biodiversity and Conservation 12: 823-836.

Trolle, M. \& M. Kéry. 2003. Estimation of ocelot density in the Pantanal using capture-recapture analysis of cameratrapping data. Journal of Mammalogy 84 (2): 607-614.

Trolle, M. \& M. Kéry. 2005. Camera-trap study of ocelot and other secretive mammals in the northern Pantanal. Mammalia 69 (3-4): 405-412.

Voss, R.S. \& L.H. Emmons. 1996. Mammalian diversity in neotropical lowland rainforests: a preliminary assessment. Bulletin of American Museum of Natural History 230: 1-115.

Wilson, D.E. \& D.M. ReEder. 2005. Mammal species of the World. A taxonomic and geographic reference. Washington, Johns Hopkins University Press, $3^{\text {rd }}$ ed., 2142p.

ZAR, J.H. 1996. Biostatistical analysis. Upper Saddle River, Prentice-Hall International, $3^{\text {rd }}$ ed., 662p.

Recebido em 05.IV.2007; aceito em 01.VIII.2007. 